\title{
Fundamental Aspects of Calciothermic Process to Produce Titanium
}

\author{
Katsutoshi Ono \\ Emeritus Professor, Kyoto University
}

Calciothermic reduction incorporated with electrolytic reclamation of calcium in a single reactor is a new method for extracting titanium from its oxide. Titanium dioxide is typically reduced in molten calcium chloride by calcium existing in this medium as soluble state, not by elemental calcium, to give also soluble calcium oxide and isolated granular sponge titanium to be recovered. This soluble calcium is reproduced simultaneously by electrolyzing the dissolved calcium oxide in the vicinity of the reduction zone of the reactor. In this paper, an outline of this advanced reactor is demonstrated especially from physico-chemical points of view with regard to the following items: (1) Thermochemistry of the $\mathrm{CaCl}_{2}$-CaO-Ca melt, (2) The electrolytic dissolution of calcium into molten calcium chloride. (3) Measure of impurities control, (4) Calciothermic reduction of titanium dioxide in molten calcium chloride.

(Received November 4, 2003; Accepted January 14, 2004)

Keywords: titanium, calcium, calciothermic reduction, titanium dioxide, calcium chloride, calcium oxide, fused salt electrolysis

\section{Introduction}

Recently, direct reduction of titanium oxide by calcium in molten salt is being given attention to produce titanium, ${ }^{1)}$ because this route would improve smelting performance, which would allow for higher productivity to meet increased demand as necessary. Direct reduction means that the processes are unified in a single reactor. Theoretically, it would be ultimate titanium extraction metallurgy. This process has been made possible particularly on the basis of the thermochemical properties that both calcium and calcium oxide exhibit large solubility in molten calcium chloride. It becomes then possible for reduction of titanium oxide and electrolysis of calcium to coexist in a common calcium chloride bath, although some portion of soluble calcium is lost by undesirable but inevitable back-reactions.

Besides the ongoing test program for experimental verification of this concept, its fundamental background should be considered and clarified. This paper was intended to address the thermodynamic basis of the reactions as well as the design basis for the reactor system. Especially as for the cell construction scheme, the cell must be built entirely with calcium chloride lining which is quite compatible with calcium-bearing salt.

\section{Description of the Process}

Raw material to be reduced is titanium dioxide synthesized from the ilmenite. The reduction process is essentially completed with a single reactor consisted of a number of unit cell arranged in parallel, and operated continuously. The base medium of this reactor is molten calcium chloride. Titanium is elaborated in the molten calcium chloride containing calcium of $3-5 \mathrm{~mol} \%$ as reducing agent and calcium oxide of $4-9$ mol\% as reduction by-product at the temperature range 1070 to $1170 \mathrm{~K}$. Titanium oxide is reduced in this medium by the reaction

$$
\mathrm{TiO}_{2}+2 \mathrm{Ca}=\mathrm{Ti}+2 \mathrm{CaO}
$$

Calcium consumed here is continuously reclaimed in the same cell from the reduction product calcium oxide dissolved

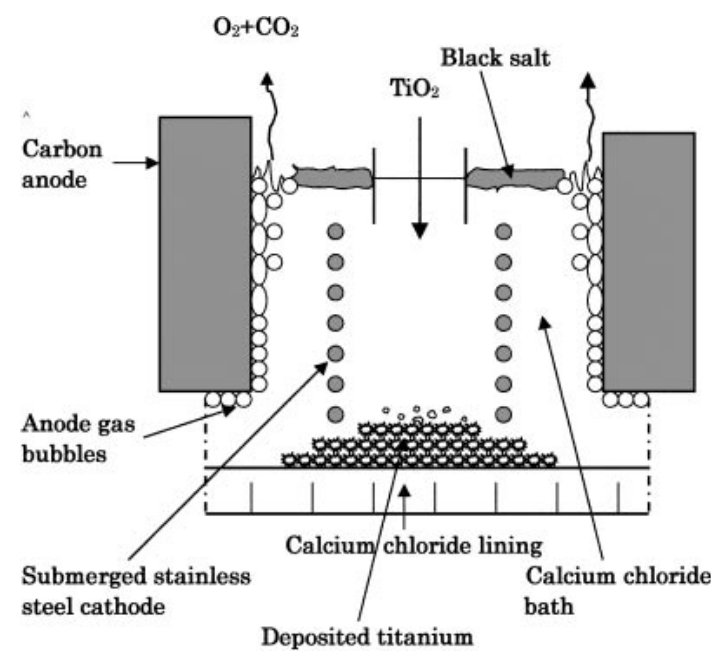

Fig. 1 Schematic diagram of the unit cell constitution.

in this medium by electrolysis using the carbon anode and the stainless steel cathode. The voltage applied is kept well beneath the decomposition voltage of calcium chloride considering the voltage losses due to the resistance of electrodes, bath and other materials used in the electric circuit. This reactor system is then characterized by that both reduction and electrolysis hold a bath in common. The process is thus a closed-loop continuous titanium elaboration, unifying the reduction with the electrolysis within unit cell as represented in Fig. 1.

The cell arrangement was selected as being simple to construct and operate, and the cell is not closed but open to the air. The inner lining of reactor is exclusively made of calcium chloride blocks for the reason of both contamination of titanium by lining material and corrosion resistance against calcium. Cathode with lattice framework is employed to make counter-diffusion of the bath constituents possible between two regions, the electrolysis and the reduction, and totally submerged in the bath. It should be installed at an appropriate level from the bottom of the cell but below the black-salt layer. Lattice framework of cathode is necessary also by another reason that the electrolytic reaction must 
occur all around the cathode surface including the side facing to the reduction region, i.e. the opposite side to the anode. The reduction region is located between two neighboring cathodes. Titanium oxide powders are subjected to reduction in suspension in the bath, and converted to metallic phase before landing on the bottom.

The black-salt is back-reaction product at the carbon anode, consisted of mixture of carbon, calcium oxide and molten calcium chloride. This agglomerated carbon and calcium oxide floats to the surface and covers the top of the bath to form a dense layer that can serve as protection against oxidation of calcium by the air.

The heat to maintain the bath at the desired temperature is supplied by the Joule heat released in the electrolytic zone, in the same manner as heat is supplied for the aluminum electrolytic process. As the zones for electrolysis and reduction are combined in the same bath, the energy issued from the exothermic reduction of titanium oxide by calcium produces additional heat. According to this analysis, theoretical requirement of electric power is approximately $3200 \mathrm{kWh} /$ tonTi, assuming that carbon anode is entirely burnt to carbon dioxide.

In view of environmental problem, it is better to enrich the reactor off-gas with oxygen. This could be improved, to some extent, by increasing anode current density and by using carbon anode with chemically less active properties.

\section{Thermochemistry of the Process}

This process is thermodynamically based on the $\mathrm{CaCl}_{2}$ $\mathrm{CaO}-\mathrm{Ca}$ ternary system at the operating temperatures between 1070 and $1170 \mathrm{~K}$ as represented in Fig. 2. Molten calcium chloride has relatively large solubility for calcium oxide. ${ }^{2)}$ The calcium ion in the stoichiometric calcium chloride is normally $\mathrm{Ca}^{2+}$. It has been recognized that some amounts of calcium dissolve in the molten calcium chloride. ${ }^{3)}$ Such a melt is no longer stoichiometric, but an equilibrium mixture of $\mathrm{Ca}^{2+}, \mathrm{Ca}^{+}, \mathrm{Cl}^{-}$ions and free electrons to maintain electroneutrality. ${ }^{3)}$ Therefore, the molten calcium chloride has a solubility for calcium, the solubility line in the ternary system being tentative. When it exceeds the solubility limit of

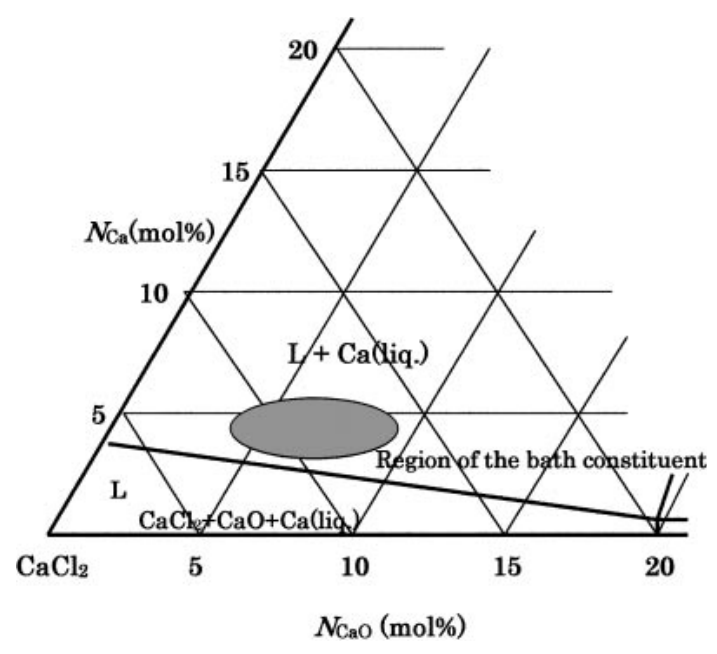

Fig. 2 Composition of the bath in the $\mathrm{CaCl}_{2}-\mathrm{CaO}-\mathrm{Ca}$ ternary system.
$\mathrm{Ca}^{+}$ion, pure calcium should appear somewhere in the bath and then its activity becomes unity. Such molten calcium chloride has a strong reducing power equivalent to pure calcium, although its basic structure is of a molten salt.

Titanium oxide powders are reduced in the working medium of molten calcium chloride with the composition indicated in Fig. 2 according to the reaction

$$
\mathrm{TiO}_{2}+2 \mathrm{Ca}^{+}+2 \mathrm{e}^{-}=\mathrm{Ti}+2 \mathrm{Ca}^{2+}+2 \mathrm{O}^{2-}
$$

If the titanium oxide particles meet the over-saturated calcium, they are also immediately reduced to the metal by the thermochemical reaction

$$
\mathrm{TiO}_{2}+2 \mathrm{Ca}=\mathrm{Ti}+2 \mathrm{Ca}^{2+}+2 \mathrm{O}^{2-}
$$

The interstitial oxygen in the reduced titanium particles, $[\mathrm{O}]_{\mathrm{Ti}}$ is subsequently removed by $\mathrm{Ca}^{+}$and calcium according to the reactions

$$
\begin{aligned}
{[\mathrm{O}]_{\mathrm{Ti}}+\mathrm{Ca}^{+}+\mathrm{e}^{-} } & =\mathrm{Ca}^{2+}+\mathrm{O}^{2-} \\
{[\mathrm{O}]_{\mathrm{Ti}}+\mathrm{Ca} } & =\mathrm{Ca}^{2+}+\mathrm{O}^{2-}
\end{aligned}
$$

The oxygen level attainable with these reactions depends on the equilibrium ratio of the activities of calcium oxide and calcium in the bath, $a_{\mathrm{CaO}} / a_{\mathrm{Ca}}$. Because the reduction byproduct calcium oxide dissolves in the bath, the reduction can be promoted by fluxing away the product oxide. Low ratio of activity would thus be achieved to eliminate the residual oxygen down to the level of 0.05 mass $\%{ }^{4)}$ The molten calcium chloride is, therefore, unique medium that exhibits thermochemical feasibility for reducing titanium oxide.

\section{Electrolytic Dissolution of Calcium into Molten Calcium Chloride}

Since calcium has a solubility for molten calcium chloride, the electrolyzed calcium leaves the cathode surface to diffuse into the molten salt without being deposited as elemental calcium on the cathode. Even if the bath is being saturated with calcium, neither solid nor liquid elemental calcium can precipitate on the free surface of cathode, at least when used a low current density of cathode. Over-saturated calcium could form clusters drifting in suspension inside the bath. A strong reducing agent, $\mathrm{CaCl}_{2}-\mathrm{CaO}-\mathrm{Ca}$ salt-mixture, is produced as a result.

On the other hand, back-reactions can occur on the anode to convert calcium in the bath into calcium oxide and carbon, caused by the anodic gases containing $\mathrm{O}_{2}$ and $\mathrm{CO}_{2}$ as shown below.

$$
\begin{aligned}
2 \mathrm{Ca}+\mathrm{O}_{2} & =2 \mathrm{CaO} \\
2 \mathrm{Ca}+\mathrm{CO}_{2} & =\mathrm{C}+2 \mathrm{CaO}
\end{aligned}
$$

These reactions produce a black-salt with dispersed carbon particles, and a continuous dense black layer is formed on the top of the salt. These side-reactions originate an inevitable fall in electrolytic current efficiency.

The current efficiency is an important factor governing not only productivity but also energy efficiency of the production cell. So, it will be useful to formulate this term with respect to the unit cell design. In view of simplicity of the following discussion, we assume that the bath is always held homogeneous in calcium, that is a complete mixing condition, and it 
is also assumed that no calcium is lost out of the working medium through the dense black-salt covering the top of the bath. Since the back-reactions take place at the interface between the anodic gas bubbles and the bath, the amount of calcium lost per unit time is represented, using the average calcium concentration in the bath $C\left(\mathrm{~mol} / \mathrm{m}^{3}\right)$, the total effective reaction area $A\left(\mathrm{~m}^{2}\right)$, and the mass transfer coefficient in the bath at bath-gas interface $k(\mathrm{~m} / \mathrm{s})$, to be $k A C(\mathrm{~mol} / \mathrm{s})$. Now, if the cell is operated under constant current, the quantity of calcium generated per unit time is invariable, denoting $\alpha(\mathrm{mol} / \mathrm{s})$. Mass balance with respect to calcium is then described as

$$
V(\mathrm{~d} C / \mathrm{d} t)+k A C-\alpha=0
$$

where $V\left(\mathrm{~m}^{3}\right)$ is total volume of the bath. By solving this equation under the initial condition, $C=0$ at $t=0$, starting point of the cell operation, we can express the calcium concentration as function of time as follows.

$$
C=(\alpha / k A)\{1-\exp [-k(A / V) t]\}
$$

Finally, the electrolytic current efficiency $\varepsilon_{\mathrm{C}}$ may be written with the aid of the eq. (9) as

$$
\varepsilon_{\mathrm{C}}=1-(k A C / \alpha)=\exp [-k(A / V) t]
$$

where the surface to volume ratio $(A / V)$ is a factor which plays an important role in determining the rate of calcium losses. The eqs. (9) and (10) are only simplifying the phenomena, but useful to consider the amount of reductant calcium saved in the bath for the reduction of titanium oxide. The eqs. (9) and (10) are shown schematically in Fig. 3 together with the values of calcium concentration and current
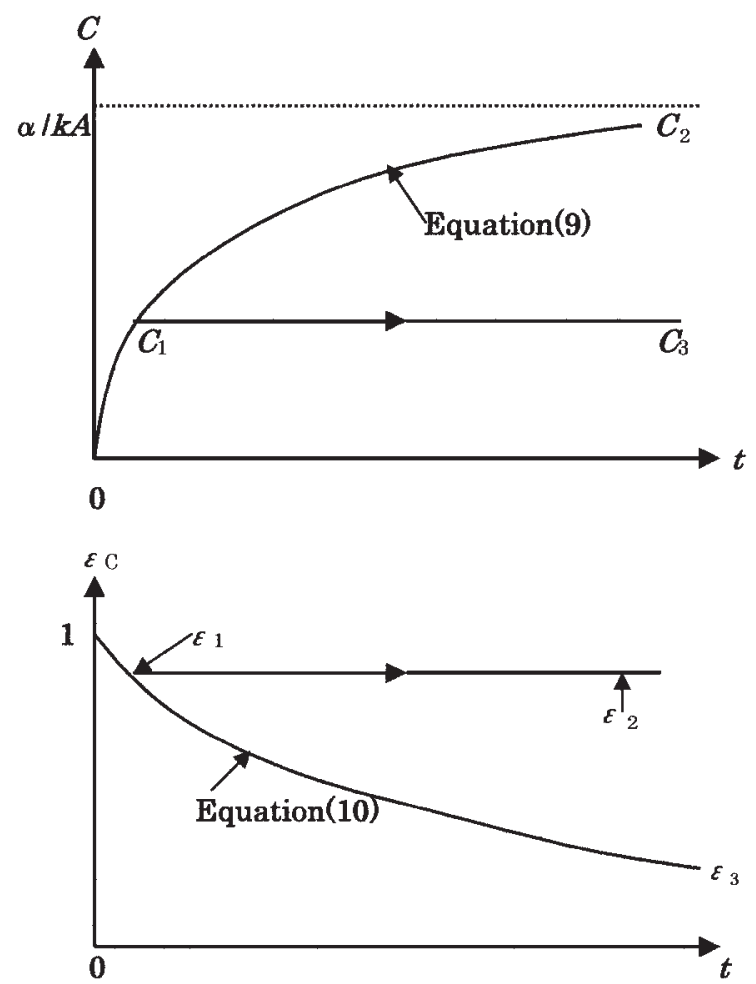

Fig. 3 Schematic representation of change in calcium concentration and electrolytic current efficiency with reduction time. efficiency if the cell is working under steady state. With a constant current, concentration of calcium increases with time according to the eq. (9)from 0 to $C_{2}$, and infinitely approaches $\alpha / k A$. Inversely, current efficiency decreases with time according to the eq. (10) from 1 to $\varepsilon_{3}$, and infinitely approaches 0 . In the practical cell operation, when the calcium concentration reaches the appropriate level $C_{1}$, about $5 \mathrm{~mol} \%$, continuous feeding of titanium oxide is started by a rate just compensated with the quantity of calcium produced by the current $\varepsilon_{1} I$ (A). Afterwards, the concentration of calcium in the bath is kept constant as $C=C_{1}=C_{3}$. Consequently, the current efficiency is also held constant as $\varepsilon_{\mathrm{C}}=\varepsilon_{1}=\varepsilon_{2}$. Note that the current efficiency is not a factor known beforehand, but it should be determined by a number of results achieved during long period of operation.

Of course, the idealized theoretical approach as described above should preferably be replaced by a real expression of kinetics in the vicinity of anode surface, but it may be useful, as a first step, to show the phenomena qualitatively. Indeed, the background of the apparent reactions is too complicated to treat mathematically. The anode gas bubbles emitted from the anode surface must rise very fast involving a turbulent flow. If we can assume that the chemical reactions between anode gas and calcium exposed to the interface follow fast kinetics, the rate-determining step for the back-reactions must be transfer of calcium from the bath to the gaseous phase. This must be due to the fact that as shown in Fig. 4, the reaction product, solid mixture of carbon and calcium oxide, could cover the bubble-salt interface, so that it must reduce not only the effective reaction area but also diffusivity of calcium from the bath toward the bubbles.

\section{Measure for Impurities Control}

Carbon, nitrogen, oxygen, silicon, and iron are commonly found as residual elements in sponge. These elements must be held to acceptably low levels, because they lower the ductility of the final product. However, difficulty to meet purity requirements for titanium may be attributed to its chemically active properties at high temperatures. Most of

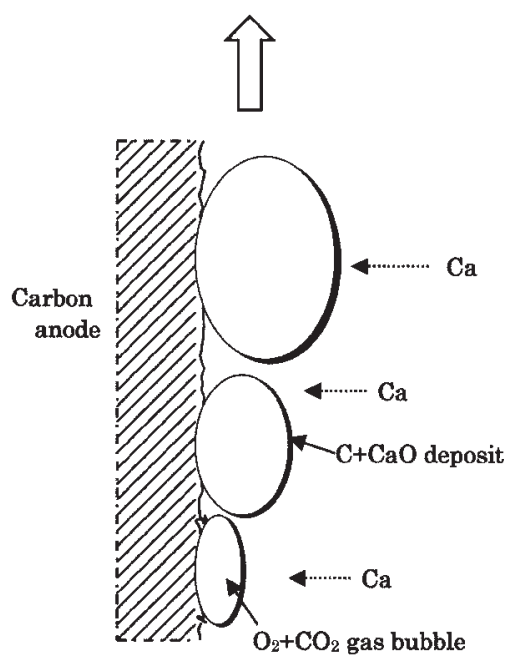

Fig. 4 Conceptual drawing of interaction between $\mathrm{Ca}$ and gas bubbles. 
the impurities in the feedstock will remain as impurities in the refined titanium. As a problem of metal processing side, in order to exclude all the risk of contamination caused by cell construction materials, the substances for cell lining must firstly be considered. Most importantly, the calcium chloride lining should exclusively be selected. Calcium and titanium can decompose almost all oxide refractory materials, and titanium can absorb immediately their constituent elements. In the same way, employment of metal lining like stainless steel also brings about serious damage to deposited titanium, because a part of electrolytic current passes through the conductive wall with continuously dissolving Fe into the bath as $\mathrm{Fe}$ ions, that is a side circuit with ineffectual detour current. Therefore, use of the calcium chloride lining is consequence of such consideration as unique solution.

Next, carbon must be taken into account so as not to enter the reduced titanium, since titanium has very strong affinity with carbon. Naturally, there is no solubility of elemental carbon for calcium chloride melt. If carbon particles are separated and leave from the surface of carbon anode, they rise to the top of the bath together with the anodic gas bubbles. Since this density separation gets along perfectly, there is practically no existence of free carbon drifting inside the bath.

Carbon dioxide has a solubility in molten calcium chloride containing calcium oxide, ${ }^{5)}$ but this is possible only if calcium is not present in the bath. If calcium is present in the bath, carbon dioxide reacts with calcium to form carbon and calcium oxide by the eq. (7) at gas-melt interface before being dissolved in the bath. Namely, it may be said there is no solubility of carbon dioxide for the $\mathrm{CaCl}_{2}-\mathrm{CaO}-\mathrm{Ca}$ melt. The anode gas bubbles are rising along the anode surface within about $10 \mathrm{~mm}$ apart from it. For prevention against contamination of reduced titanium by carbon, it should preferably be prescribed that titanium particles must not be entered into the zone with thickness of $50 \mathrm{~mm}$ just in the neighborhood of the bubbles as well as just beneath the black-salt.

Regarding to silicon and iron, impurity levels of these elements must be controlled in the ore preparation step to synthesize the titanium oxide, because there is no way to remove silicon and iron from titanium even with this reactor.

\section{Calciothermic Reduction of Titanium Oxide in Mol- ten Calcium Chloride}

In general, defect structure of oxide has significant relation to its high-temperature oxidation and reduction. Kofstad ${ }^{6)}$ proposed that defect structure of titanium dioxide comprises doubly charged oxygen vacancies and interstitial ions with three and four effective charges. In this case, calciothermic reduction proceeds by diffusion mechanism inside the grains, i.e. diffusion of $\mathrm{O}^{2-}$ ions toward exterior and/or $\mathrm{Ti}^{4+}$ ions toward interior of a titanium dioxide particle, which determines the rate of reduction. This rate-determining step may predict that the reduction becomes faster as the particle size is smaller. The titanium dioxide powders as received are desirably so fine as $0.5 \mu \mathrm{m}$ in average as seen in Fig. 5.

The titanium oxide powders are directly fed to the cell by inserting into the clean reduction zone of the bath. They are transformed to metallic phase in course of sinking slowly to the bottom, agglomerated each other prior to sintering, and these particles subsequently begin to adhere and grow together with establishing grain boundaries and metastable pores. Even after landing on the bottom, grain growth continues, and the cross sectional areas of the pore decrease. Morphology of grains at the final stage is also shown in Fig. 5.

In parallel with that sintering process, chemical diffusion process is taking place inside the grains. Diffusion of the
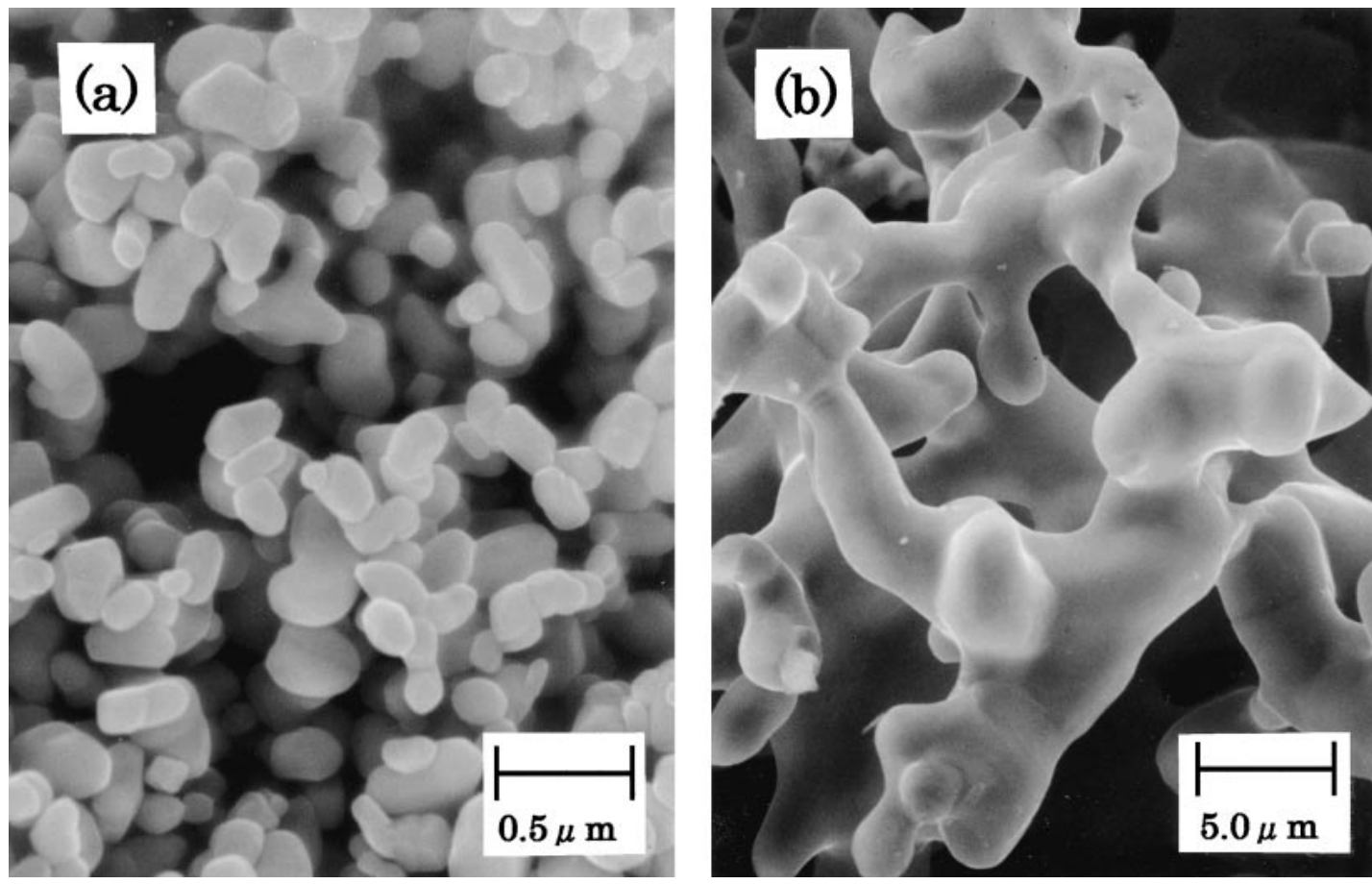

Fig. 5 SEM micrographs of the $\mathrm{TiO}_{2}$ powders as received (a) and the titanium reduced at $1173 \mathrm{~K}$ for 6 hours (b). 
interstitial oxygen governs the kinetics of the reaction for its elimination, so that it needs a fairly long time to lower the oxygen concentration down to levels below 0.08 mass $\%$.

\section{Conclusion}

The single-cell elaboration of titanium, in which the direct oxide reduction and the direct electrolytic recovery of the reducing agent are combined homogeneously and continuously, will open the way to benefits for both energy and productivity.

The advanced fundamental understanding and the identification of problems are expected to lead to a new program of investigation using a single pilot cell facility operated continuously.

\section{REFERENCES}

1) G. Z. Chen, D. J. Fray and T. W. Farthing: NATURE 407 (2000) 361364.

2) D. A. Wenz, I. Johnson and R. D. Wolson: J. Chem. Eng. Data 14(2) (1969) 250-252.

3) V. Dosaj, C. Aksaranan and D. R. Morris: J. Chem. Soc., Faraday Trans. 71 (1975) 1083-1098.

4) T. H. Okabe, R. O. Suzuki, T. Oishi and K. Ono: Tetsu-to-Hagane 77(1) (1991) 93-99.

5) M. Maeda and A. McLean: Trans. Iron Steel Soc. 8 (1987) 23-29.

6) P. Kofstad: Nonstoichiometry, diffusion, and Electrical Conductivity in Binary Metal Oxides, (Wiley-Interscience, A Division of John Wiley \& Sons, Inc. New York, 1972) p. 139. 